\title{
Nonmelanoma cutaneous cancers involving the skull base: outcomes of aggressive multimodal management
}

\author{
Shaan M. Raza, MD, ${ }^{1}$ Rohan Ramakrishna, MD, ${ }^{1}$ Randal S. Weber, MD, ${ }^{2}$ \\ Michael E. Kupferman, MD, ${ }^{2}$ Paul W. Gidley, MD, ${ }^{2}$ Ehab Y. Hanna, MD, ${ }^{2}$ and Franco DeMonte, MD' \\ Departments of ${ }^{1}$ Neurosurgery and ${ }^{2} \mathrm{Head}$ and Neck Surgery, The University of Texas MD Anderson Cancer Center, \\ Houston, Texas
}

\begin{abstract}
OBJECT A relative paucity of information exists regarding outcomes from craniofacial resection for advanced nonmelanoma skin cancers involving the skull base. In light of advances in surgical technique and adjuvant therapy protocols, the authors reviewed their surgical experience to determine disease control rates, overall survival (OS), morbidity, and mortality.

METHODS A retrospective review of 24 patients with nonmelanoma cutaneous cancers with skull base involvement treated with craniofacial resection at The University of Texas MD Anderson Cancer Center from 1994 to 2012 was performed. Of these patients, 19 (79\%) had squamous cell carcinoma (SCC), 4 (17\%) had basosquamous carcinoma (BSCC), and 1 patient (4\%) had adenocarcinoma. Factors as assessed were prior treatment, TNM staging, tumor involvement, extent of intracranial extension, margin status, postoperative complications, recurrence, disease status at last follow-up, and long-term survival. The majority of tumors were T4 (67\%) according to the TNM classification; perineural extension was noted in $58 \%$, cavernous sinus involvement in $25 \%$, and dural involvement in $29 \%$.
\end{abstract}

RESULTS Postoperative complications occurred in 4 patients (17\%) including 1 death. Kaplan-Meier estimates were calculated for OS and progression-free survival (PFS). Median OS was 43.2 months with an $82 \% 1$-year OS and $37 \%$ 5 -year OS; the median PFS was 91.2 months. Margin status was positively associated with median OS in SCC (91 months [for negative margins] vs 57 months, $p=0.8$ ) and in BSCC (23.7 vs 3.2 months, $p<0.05$ ). Postoperative radiotherapy was associated with improved median OS ( 43.2 vs 22 months, $p=0.6)$. Brain involvement was uniformly fatal after 1 year, while cavernous sinus involvement (31 vs 43 months, $p=0.82$ ), perineural disease ( 31 vs 54 months, $p=$ 0.30 ), and T4 stage (22 vs 91.2 months, $p=0.09$ ) were associated with worsened OS. Similar associations were found with median PFS.

CONCLUSIONS Aggressive multimodality management with surgery and postoperative radiotherapy can positively impact locoregional control and OS. With improvements in technique and adjuvant therapy protocols, treatment can still be considered in situations of perineural disease and cavernous sinus involvement and as a salvage option for patients in whom prior treatment has failed. As patients with advanced NMSCs often have few options, craniofacial resection, as part of a coordinated multimodal management plan, is justified if it can be performed safely.

http://thejns.org/doi/abs/10.3171/2014.10.JNS141037

KEY WORDS malignancy; cavernous sinus; perineural disease; craniofacial resection; skull base; skin cancer; oncology

$\mathrm{R}$ EPRESENTING the most common human neoplasm, skin cancers occur in the head and neck region in nearly $90 \%$ of cases due to sun exposure. Of these, nonmelanoma skin cancers (NMSCs) pose a unique set of challenges. Basal cell carcinoma (BCC) is the most common malignancy in this area, with squamous cell carcinoma (SCC) second in frequency. BCC is characterized by its propensity for local aggressive growth and the capacity to reach large sizes (greater than $2 \mathrm{~cm}$ ). SCC is the second most common cutaneous malignancy and is differentiated from BCC by its tendency to spread to regional lymph nodes ( $15 \%$ in tumors greater than $2 \mathrm{~cm}$ ) and for perineural invasion $(2 \%-15 \%)$. While considered a histological subtype of BCC, basosquamous carcinoma (BSCC) is

ABBREVIATIONS $B C C=$ basal cell carcinoma; $B S C C=$ basosquamous cell carcinoma; $C N=$ cranial nerve $;$ MDACC $=$ MD Anderson Cancer Center; NMSC $=$ nonmelanoma skin cancer; OS = overall survival; PFS = progression-free survival; $S C C=$ squamous cell carcinoma.

SUBMITTED May 8, 2014. ACCEPTED October 20, 2014.

INCLUDE WHEN CITING Published online April 24, 2015; DOI: 10.3171/2014.10.JNS141037.

DISCLOSURE The authors report no conflict of interest concerning the materials or methods used in this study or the findings specified in this paper. 
thought to be more aggressive with a significantly increased incidence of local and distant metastases $(5 \%){ }^{2}$ The primary treatment for patients with NMSCs remains surgical excision with negative pathological margins.

Skull base and intracranial involvement can occur via several mechanisms in addition to direct invasion of the underlying soft tissue and calvaria. The motor and sensory nerves of the facial viscera provide an important conduit for perineural spread in intracranial invasion. Perineural involvement is unfortunately common in advanced cutaneous malignancies, occurring in up to $24 \%$ of cases. ${ }^{4}$ Additionally, NMSCs are notorious for infiltration along perichondrium, periosteum, and the vasculature. ${ }^{12}$ Consequently, active disease can remain in these areas if it is not addressed during surgical removal and can be a source of recurrences after multiple attempts at surgical extirpation. As a result, skull base involvement complicates a surgical strategy to obtain margins and is an important consideration in obtaining acceptable surgical and oncological outcomes.

There is a paucity of data in the neurosurgical literature regarding the role of multidisciplinary treatment and the expected impact on locoregional control and overall survival specifically with NMSCs with skull base and/or intracranial invasion. While the surgical objective of obtaining negative margins is documented in the literature, the extent to which surgery should be pursued in light of varying degrees of skull base, intracranial, and perineural invasion warrants study. An understanding of these factors would guide any surgical strategy. Additionally, inconsistencies in the administration of chemotherapy and radiation therapy with advanced NMSCs and expected impact on locoregional and systemic disease control warrant an analysis. We previously reported our experience with a cohort of 35 patients who underwent surgery from 1982 through 1993. ${ }^{1}$ In light of surgical advancements and shifts in adjuvant therapy protocols, we reviewed our experience from 1994 through 2012; primary outcomes assessed were overall survival (OS), progression-free survival (PFS), and short-term surgical outcomes.

\section{Methods}

A retrospective chart review of all patients evaluated at The University of Texas MD Anderson Cancer Center (MDACC) for NMSC involving the skull base and treated via craniofacial resection between 1994 and 2012 was performed. Patients were identified via a search of the prospectively collected Head and Neck Tumor Registry Database. This study was performed under an institutional review board-approved protocol in compliance with the regulations set by our institution for the study of human subjects. All treatment decisions were made in a multidisciplinary fashion with input from radiation oncology, medical oncology, neurosurgery, head and neck surgery, and plastic surgery. The goal of our surgical strategy was for safe gross-total resection with negative margins, while all reconstructive flaps were designed with the intent to provide vascularized dural coverage and separation of the intracranial space. Clinical charts, operative records, preoperative and postoperative imaging findings (MRI and/or CT), pathology (including margins), and postop- erative documentation were noted. In addition to demographic data, information was collected regarding prior treatments, clinical and pathological data, complications, and outcomes. A univariate analysis was performed using SPSS software to determine predictors of PFS and OS; a multivariate analysis was not performed due to the limited power of the study. Kaplan-Meier estimates of PFS and OS were calculated, and survival curves were compared using the log-rank test. A p value $<0.05$ was considered significant. The small number of disease-related deaths in each subcohort prohibited the use of disease-specific survival for statistical analysis.

\section{Results}

\section{Patient Demographics}

Twenty-four patients, ranging from 42 to 83 years in age (median 67 years), had histological confirmation of NMSC and underwent treatment at MDACC (Table 1) for skull base involvement. The most common presenting symptoms were an ulcerated nonhealing wound (38\%) and trigeminal facial pain $(29 \%)$. A majority of patients $(62 \%)$ presented to MDACC with recurrences after prior treatment (Table 2). The median time from last treatment to recurrence or presentation at MDACC was 9.1 months. Of note, 11 patients $(46 \%)$ had received some form of radiation therapy prior to our intervention, and, as a consequence of prior treatment fields, these patients were ineligible to receive postoperative radiotherapy after our intervention.

\section{Tumor Characteristics}

Nineteen (79\%) primary tumors were SCC, 4 (17\%) were BSCC, and 1 (4\%) was an adenocarcinoma (Table 3). Of the SCCs, 7 were poorly differentiated, 8 were moderately differentiated, and 4 were well differentiated.

At the time of surgery, a majority of patients presented with T4 tumors (67\%), and no patients had lymph node disease, systemic disease, or leptomeningeal spread. With regard to involvement of extracranial structures, tumors involved the orbit (54\%), maxillary sinus (46\%), infratemporal fossa $(33 \%)$, nasal cavity $(21 \%)$, and ethmoid sinus (17\%). In those patients with orbital involvement, the periorbita and orbital contents were invaded in all patients.

Sites of cranial base extension included the anterior fossa only $(25 \%)$, middle fossa only $(58 \%)$, and both the anterior and middle fossae (17\%). Twenty-five percent of the cohort had involvement of the petrous component of the temporal bone. A significant fraction of patients (29\%) had dural involvement, while 2 patients had evidence of subdural tumor at the time of surgery. Additionally, 25\% had tumor extension into the cavernous sinus present either on preoperative imaging or noted based on intraoperative findings. With regard to perineural involvement, cranial nerves (CNs) V (46\%) and VII (12.5\%) were the most commonly involved; involvement ranged from infiltration of named distal branches to the main trunk.

\section{MDACC Treatment Strategies}

Treatment strategies were determined based on multidisciplinary consensus during the study period and are 
TABLE 1. Demographic data

\begin{tabular}{lc}
\hline \multicolumn{1}{c}{ Variable } & Value $^{*}$ \\
\hline Age $(\mathrm{yrs})$ & 67 \\
\hline Median & $42-83$ \\
\hline Range & $5(21)$ \\
\hline Sex & $19(79)$ \\
\hline Male & $14(58)$ \\
\hline Female & $7(29)$ \\
\hline Comorbidities & $4(17)$ \\
\hline Cardiac & $1(4)$ \\
\hline Other primary cancer & $1(4)$ \\
\hline Endocrine & $1(4)$ \\
\hline Neurological & \\
\hline Psychiatric & $10(42)$ \\
\hline Presenting symptoms & $6(25)$ \\
\hline Ulcerated/nonhealing wound & $3(12.5)$ \\
\hline Facial pain & $2(8)$ \\
\hline Facial nerve palsy & $1(4)$ \\
\hline Visual decline & $1(4)$ \\
\hline Hearing loss & 90 \\
\hline Proptosis & \\
\hline Median Karnofsky Performance Scale score & \\
\hline
\end{tabular}

* Values indicate the number of patients (\%) unless specified otherwise.

summarized in Table 4. Of this cohort, 3 patients had gross invasion of the facial nerve, prompting sacrifice proximally from the tympanic and/or mastoid segments of the nerve to the distal-most branches after a parotidectomy had been completed. Two patients underwent immediate facial nerve reanimation with cable nerve graft in addition to static reconstructive techniques (i.e., tensor fascia lata sling and gold weights). Microvascular free tissue reconstructions consisted of rectus flaps (12 patients), anterolateral thigh flaps (5 patients), forearm flap (3 patients), and latissimus dorsi flap (1 patient).

A majority of patients underwent surgery followed by adjuvant radiotherapy (54\%); adjuvant chemoradiation therapy was used in 1 patient. Patients who had received radiation prior to our treatment were ineligible for reirradiation due to prior fields.

Postoperative radiation therapy was in the form of intensity-modulated radiation therapy or proton therapy with a mean dose of $60 \mathrm{cGy}$ to the entire preoperative tumor bed volume; elective neck irradiation was not performed in any patient. Stereotactic radiosurgery was used to control known positive margins within the cavernous sinus or cranial nerves in 1 patient who did not receive any other form of postoperative radiotherapy. One patient with recurrent SCC after prior surgery and radiation therapy underwent postoperative stereotactic radiosurgery with 20 Gy to a single positive margin within the cavernous sinus after having undergone a gross-total resection with otherwise negative margins.

\section{Surgical Outcomes}

Gross-total resection was achieved in all but 1 patient
TABLE 2. Characteristics of treatment received prior to presentation at MDACC $(n=15)^{*}$

\begin{tabular}{lc}
\hline \multicolumn{1}{c}{ Prior Treatment } & No. of Patients (\%) \\
\hline Surgery only & $4(17)$ \\
\hline Surgery \& radiation & $9(38)$ \\
\hline Surgery, chemotherapy, \& radiation & $1(4)$ \\
\hline Biopsy \& radiation & $1(4)$ \\
\hline
\end{tabular}

* Median time from last treatment to presentation at MDACC $=9.1$ months.

(Table 5). Microscopically negative margins were obtained in $62 \%$ of patients and gross-total resection with positive microscopic margins in 33\%. The most common site of positive margins was within the cavernous sinus (50\% of those with positive margins); another common site of positive disease was the middle fossa dura medial to the gasserian ganglion ( $25 \%$ of those with positive mar-

TABLE 3. Disease characteristics

\begin{tabular}{|c|c|}
\hline Tumor Characteristics & No. of Patients (\%) \\
\hline \multicolumn{2}{|l|}{ Histology } \\
\hline SCC & $19(79)$ \\
\hline BSCC & $4(17)$ \\
\hline Adenocarcinoma & $1(4)$ \\
\hline \multicolumn{2}{|l|}{ Epicenter } \\
\hline Forehead & $8(33)$ \\
\hline Cheek & $5(21)$ \\
\hline Temple & $5(21)$ \\
\hline Ear (external ear or external auditory meatus) & $4(17)$ \\
\hline Medial canthus & $1(4)$ \\
\hline Nasal ala & $1(4)$ \\
\hline \multicolumn{2}{|l|}{ Facial involvement } \\
\hline Orbit & $13(54)$ \\
\hline Maxillary sinus & $11(46)$ \\
\hline Infratemporal fossa & $8(33)$ \\
\hline Nasal cavity & $5(21)$ \\
\hline Ethmoid sinus & $4(17)$ \\
\hline \multicolumn{2}{|l|}{ Cranial skull base involvement } \\
\hline Anterior fossa & $6(25)$ \\
\hline Middle fossa & $14(58)$ \\
\hline Anterior \& middle fossa & $4(17)$ \\
\hline Temporal bone & $6(25)$ \\
\hline \multicolumn{2}{|l|}{ Extent of cranial/skull base involvement } \\
\hline Bone & $24(100)$ \\
\hline Dura & $7(29)$ \\
\hline Subdural/brain & $2(8)$ \\
\hline Cavernous sinus & $6(25)$ \\
\hline \multicolumn{2}{|l|}{$\mathrm{CN}$ involvement } \\
\hline $\mathrm{CN} \mathrm{V}$ & $11(46)$ \\
\hline CN VII & $3(12.5)$ \\
\hline \multicolumn{2}{|l|}{ Tumor classification (stage) } \\
\hline T3 & $8(33)$ \\
\hline $\mathrm{T} 4$ & $16(67)$ \\
\hline
\end{tabular}


TABLE 4. Overall MDACC treatment strategy

\begin{tabular}{cc}
\hline \multicolumn{1}{c}{ Treatment } & No. of Patients (\%) \\
\hline Surgical approach & \\
\hline Orbital exenteration & $13(54)$ \\
\hline Transfacial approach only & $6(25)$ \\
\hline Craniofacial approach & $12(50)$ \\
\hline Lateral skull base resection & $6(25)$ \\
\hline Type of reconstruction & $21(88)$ \\
\hline Free flap & $2(8)$ \\
\hline Rotational flap & $1(4)$ \\
\hline Primary closure & $1(4)$ \\
\hline Chemotherapy & $2(8)$ \\
\hline Adjuvant & $1(4)$ \\
\hline Neoadjuvant & $1(4)$ \\
\hline Neoadjuvant \& adjuvant & $13(54)$ \\
\hline Radiation therapy & $1(4)$ \\
\hline Neoadjuvant &
\end{tabular}

gins). The 30-day complication rate was $17 \%$ (Table 5). There was 1 death in a 78-year-old patient with coronary artery disease and chronic obstructive pulmonary disease who died on postoperative Day 10 after developing pneumonia and subsequent respiratory failure.

\section{Overall Survival}

The median follow-up period for the cohort was 85 months. At the conclusion of study follow-up, 5 patients (21\%) were alive; of those patients, 4 had no evidence of disease while 1 had regional recurrence. Of those patients who died (19 patients, 79\%), 7 died with evidence of active disease (29.2\%). Twelve deaths were due to other causes or undeterminable causes. The median disease-specific survival for the cohort was 148 months.

The median OS of the cohort was 43.2 months (95\% CI 18.8-91.2 months). The 1- and 5-year OSs for all patients were $82 \%$ and $37 \%$, respectively (Table 6 ). The histological diagnosis of BSCC was associated with a trend in decreased OS in comparison with SCC (23.8 months [for BSCC] vs 53.8 months). Irrespective of histology, advanced T-stage (3 vs 4) was indicative of a poorer OS (91.2 months [for T-stage 3] vs 22.0 months). Furthermore, intracranial involvement with brain invasion was uniformly fatal within 2 years (median survival 43.2 months [for no brain invasion] vs 22.0 months, 1 -year OS $81 \%$ vs $100 \%$, 2 -year OS $62 \%$ vs $0 \%$ ). A similar trend was noted for perineural disease where median survival (53.8 months [for no perineural disease] vs 31.0 months) and 1-year survival ( $100 \%$ vs $71 \%$ ) were noted; this discrepancy was mitigated at 5 years posttreatment ( $44 \%$ vs $31 \%$ ). Adjuvant radiotherapy positively impacted median OS and 1-year OS (43.2 months [for adjuvant radiotherapy] vs 22.0 months and $92 \%$ vs $60 \%$ ); this survival benefit was mitigated by 5 years. Margin status conferred a benefit on median OS survival in SCC (91.2 months [for negative margins] vs
TABLE 5. Short-term (30-day) surgical outcomes

\begin{tabular}{cc}
\hline Outcome & No. of Patients (\%) \\
\hline Extent of resection & \\
\hline Gross-total resection & $15(62)$ \\
\hline Negative margins & $8(33)$ \\
\hline Positive margins & $1(4)$ \\
\hline Subtotal resection & \\
\hline Hospital length of stay (days) & 7 \\
\hline Median & $4-30$ \\
\hline Range & $4(17)$ \\
\hline 30-day complications & $1(4)$ \\
\hline Flap breakdown & $1(4)$ \\
\hline CSF fistula & $1(4)$ \\
\hline Hematoma & $1(4)$ \\
\hline Medical mortality
\end{tabular}

57.0 months) and median OS in BSCC (23.7 months [for negative margins] vs 3.2 months).

\section{Recurrence and Progression-Free Survival}

Forty-two percent of patients experienced disease recurrence at a median of 20 months (range 2-105 months) after the index surgery. One patient with poorly differentiated SCC experienced nodal and pulmonary metastases. The median PFS for the overall cohort was 91.2 months (Table 6). Median PFSs of 105.3 months and 16.8 months were noted for SCC and BSCC, respectively $(\mathrm{p}<0.05)$. Extent of intracranial invasion, primarily with brain invasion, was associated with a significantly worse PFS ( 7.5 for brain invasion vs 105.4 months, $\mathrm{p}<0.05$ ). Of the 6 patients with cavernous sinus disease, local recurrence occurred in 3 patients and was associated with a strong trend toward diminished PFS (105.4 for no cavernous sinus disease vs 25.5 months). T-stage (3 vs 4 ) was associated with similar decrease in PFS (91.2 for T-stage 3 vs 21.4 months); however, involvement of any specific anatomical compartment was not associated with any specific trend. When assessed histologically, the presence of negative margins was associated with a strong positive impact on mean PFS in SCC (135 months [for negative margins] vs 80 months) and BSCC (16.8 vs 3.2 months). Lastly, the use of adjuvant radiotherapy with $60 \mathrm{cGy}$ demonstrated a strong positive impact on local control after index treatment at MDACC (105.4 for adjuvant radiotherapy vs 25.5 months).

With regard to treatment for recurrences, treatment algorithms included repeat resection with chemotherapy (2 patients), chemotherapy alone (2 patients), repeat resection with radiotherapy (1 patient), and resection alone (1 patient); 4 patients refused further treatment. Treatment for recurrence was positively associated in a statistically significant fashion with OS $(\mathrm{p}<0.001)$.

\section{Discussion}

Advanced nonmelanoma cutaneous cancers of the head and neck with invasion of the skull base are rare. While much of the craniofacial literature addresses paranasal si- 
TABLE 6. Factors affecting PFS and OS*

\begin{tabular}{|c|c|c|c|c|c|c|}
\hline Covariate & Median PFS in Mos $(95 \% \mathrm{Cl})$ & $p$ Value & Median OS in Mos $(95 \% \mathrm{Cl})$ & p Value & $1-Y r$ OS & $5-Y r O S$ \\
\hline All patients & $91.2(9.6-201.1)$ & & $43.2(18.8-91.2)$ & & $82 \%$ & $37 \%$ \\
\hline Tumor histology & & 0.04 & & 0.22 & & \\
\hline BSCC & $16.8(4.2-29.7)$ & & $23.8(18.8-43.2)$ & & $100 \%$ & $0 \%$ \\
\hline SCC & $105.3(9.5-201.3)$ & & $53.8(16.5-97.1)$ & & $78 \%$ & $44 \%$ \\
\hline Temporal bone & & 0.5 & & 0.63 & & \\
\hline No & $91.2(26.6-156)$ & & $36.5(16.5-57.1)$ & & $75 \%$ & $31 \%$ \\
\hline Yes & $105.3(9.5-201.3)$ & & $31.0(18.8-203.1)$ & & $100 \%$ & $50 \%$ \\
\hline Dura & & 0.83 & & 0.32 & & \\
\hline No & $105.4(78-132.8)$ & & $23.8(13.5-91.2)$ & & $75 \%$ & $38 \%$ \\
\hline Yes & $29.6(21.0-38.3)$ & & $43.2(31.0-203.1)$ & & $100 \%$ & $42 \%$ \\
\hline Brain & & 0.04 & & 0.55 & & \\
\hline No & $105.4(19.6-191.2)$ & & $43.2(18.8-91.2)$ & & $81 \%$ & $38 \%$ \\
\hline Yes & 7.5 & & 22.0 & & $100 \%$ & $0 \%$ \\
\hline Cavernous sinus & & 0.54 & & 0.82 & & \\
\hline No & $105.4(19.6-191.2)$ & & $43.2(18.8-91.2)$ & & $88 \%$ & $38 \%$ \\
\hline Yes & $25.5(10.7-40.3)$ & & $31.0(4.2-57.1)$ & & $67 \%$ & $33 \%$ \\
\hline Perineural disease & & 0.37 & & 0.30 & & \\
\hline No & $105.4(10.4-200.4)$ & & $53.8(23.8-91.2)$ & & $100 \%$ & $44 \%$ \\
\hline Yes & $91.3(0-209)$ & & $31.0(10.0-57.1)$ & & $71 \%$ & $31 \%$ \\
\hline T-stage & & 0.38 & & 0.09 & & \\
\hline 3 & $91.2(0-183.5)$ & & $91.2(43.2-91.2)$ & & $100 \%$ & $57 \%$ \\
\hline 4 & $21.4(0-96.7)$ & & $22(13.5-36.5)$ & & $74 \%$ & $27 \%$ \\
\hline Adjuvant radiotherapy & & 0.5 & & 0.6 & & \\
\hline No & $25.5(0-108.4)$ & & $22.0(0.0-133.4)$ & & $60 \%$ & $37 \%$ \\
\hline Yes & $105.4(0-215.9)$ & & $43.2(16.5-70)$ & & $92 \%$ & $31 \%$ \\
\hline Margin status (BSCC) & & 0.04 & & 0.04 & & \\
\hline Positive & 3.2 & & 3.2 & & $0 \%$ & $0 \%$ \\
\hline Negative & $16.8(4.1-29.6)$ & & $23.7(6.3-41.2)$ & & $75 \%$ & $0 \%$ \\
\hline Margin status (SCC) & & 0.81 & & 0.80 & & \\
\hline Positive & $80(43.6-117.3) \dagger$ & & $57.0(31.3-104.5)$ & & $71 \%$ & $43 \%$ \\
\hline Negative & $135.7(70.4-201.1) \dagger$ & & $91.2(47.8-154.3)$ & & $82 \%$ & $43 \%$ \\
\hline
\end{tabular}

nus disease, there is a paucity of data that analyzes the effectiveness of surgical intervention with this disease population. Furthermore, published literature to date does not specifically focus on NMSCs with intracranial extension. ${ }^{1,9}$ Hence, the role of surgery and the extent to which it should be pursued in light of intracranial and/or cranial nerve invasion is unclear due to the relative lack of evidence on this subject. We currently pursue resection in patients who meet the following criteria: lack of major medial comorbidities, high performance status, lack of major vessel (arterial or venous) involvement, absence of lower cranial nerve involvement, lack of extensive or eloquent brain invasion, and presence of only local disease at presentation. Additionally, factors that affect progression and OS in this cohort of patients with advanced disease are unclear. The current study presents a cohort of 24 patients with NMSCs with skull base invasion; the goal of this study was to assess OS, PFS, and short-term surgical outcomes. While the study is limited by its retrospective design and small cohort, the results demonstrate several interesting trends.

\section{Impact of Multimodality Treatment of NMSCs on PFS and OS}

The data support the role of surgery with regard to PFS and OS when part of a multidisciplinary treatment regimen. Given that there was only 1 incident of distant metastases in this cohort, the goal of treatment is primarily to achieve locoregional control. Where the intended surgical outcome was for gross-total resection with negative margins, the locoregional recurrence rate was $39 \%$, the median PFS was 105 months, and the 5-year OS was $37 \%$. Additionally, $67 \%$ of the cohort had no evidence of disease at last follow-up. It is evident that once local control is obtained, a majority of patients will likely die of other 
causes with no evidence of disease, hence the discrepancy in PFS and OS.

While the extent to which resection should be pursued in all NSMCs is unclear, our data support a role for obtaining negative margins in patients with SCC and BSCC. Both mean PFS (135 vs 80 months) and median OS (91 vs 57 months) were positively impacted by a microscopically complete resection in patients with SCC. A similar, but statistically significant, trend was seen in patients with BSCC where the median OS was 23.7 months with negative margins versus 3.2 months with positive margins; however, these data should be interpreted with caution given the small numbers in each subcohort. Additionally, there were no patients with BCC in our cohort; hence, these data do not provide any guidance to their surgical management, although previous work supports a similar management plan. ${ }^{4}$ Nonetheless, our data are supportive of radical safe resection for NMSCs in general.

In comparison with our previously published cohort, our data suggest that adjuvant radiotherapy is increasingly being used in our management of advanced NMSC. ${ }^{1}$ Radiation therapy was delivered either in the form of intensitymodulated radiotherapy or proton beam radiotherapy with a dose of 60 Gy. Long-term PFS and OS are favorable if radiotherapy is administered in the postoperative setting. In particular, postoperative radiotherapy is demonstrated by our data to improve local control and OS after resection in patients with cavernous sinus involvement (median OS 57.1 vs 4.1 months), perineural disease (median OS 57.1 vs 3.3 months), and positive margins (median OS 57.1 vs 4.1 months). The positive impact of radiotherapy on local control and disease-specific survival in the management of SCCs with perineural invasion has been demonstrated in several retrospective studies.$^{6,8}$ In the context of this literature, our data indicate that resection can be considered in these circumstances if it can be performed safely in conjunction with postoperative radiotherapy. Furthermore, this is important in patients presenting with local progression after initial resection; the previous administration of radiotherapy limits the indications for surgery in situations of salvage treatment. With regard to neoadjuvant and adjuvant chemotherapy, we cannot draw any conclusions based on our data due to the extremely small numbers. However, extrapolating from the head and neck squamous cell carcinoma data, there may be a role for adjuvant chemoradiotherapy in the select group of patients with advanced local disease; ${ }^{14}$ recent Phase II studies have additionally explored the use of endothelial growth factor receptor inhibitors.?

Given the fact that all our patients had skull base invasion, it is difficult to compare these data with the handful of reports that have been published. This is further complicated by differences in outcomes that are assessed, their reporting, and the histological makeup of the reported study cohorts. In a collective multiinstitutional retrospective study, the International Collaborative Study Group assessed outcomes in a cohort of 120 patients. ${ }^{9}$ Where $34 \%$ of the cohort did not have intracranial extension, the 5-year OS and PFS were 64\% and 60\%, respectively. Ducic et al. reported a retrospective review of 21 patients with aggressive SCCs who underwent craniofacial resection. While their intended goal was for gross-total resec- tion, their extent of resection was not reported. Their cohort's PFS was reported to be $67.7 \%$ at 4 years. ${ }^{5}$ Reporting their experience with a cohort of 31 patients $(71 \%$ SCCs, $23 \%$ BCCs, and 6\% other histologies), Papadopoulos et al. demonstrated a mean PFS of 35 months. ${ }^{13}$ However, that study excluded patients with cavernous sinus or subdural invasion, and the authors' use of adjuvant therapy was not clearly delineated.

Our previous report of 35 patients (20 SCCs and 15 BCCs) was also designed to assess prognostic factors, local control rates, disease-specific survival, and complication rates. ${ }^{1}$ Long-term disease-specific survival was $20 \%$, and $11.4 \%$ of the patients were living with disease at a mean follow-up of 47.4 months. With a mean follow-up of 83 months and long-term disease-specific survival of $71 \%$, our most recent cohort has experienced improved survival. This difference in outcomes could be related to the increased administration of postoperative radiotherapy in our modern cohort (54\% vs $28.6 \%$ ). Additionally, we have perhaps refined our selection of candidates for surgeryone patient in the original cohort had disease in the cervical lymph nodes versus none in this series. Furthermore, our technique has improved as the surgical complication rate has dropped from $37.1 \%$ to $17 \%$ with an improved rate of negative margins from $51.4 \%$ to $63 \%$. The reduction in surgical morbidity along with improvements in local control and survival seen with time between the 2 studies provides positive reinforcement for the current treatment paradigms.

\section{Role of Salvage Treatment}

The role of aggressive management as a salvage treatment in patients previously treated with surgery (primarily wide excisions) with or without adjuvant chemoradiation therapy is supported by our data. Even in those patients who experienced progression despite prior therapy (at a mean of 9.1 months prior to presentation to MDACC with recurrence), salvage treatment improved mean PFS to 99.9 months (95\% CI 51.2-148.7 months). Resection at our institution changed tumors that had positive margins to those that had negative margins in many of these patients (60\%), further supporting consideration of surgery in select patients who have undergone prior treatment. The contraindications for a repeat resection include inoperable recurrence (i.e., disease primarily in the cavernous sinus), lymph node or distant metastases, or recurrence where negative margins cannot be obtained and adjuvant therapy options are not available.

Tumor recurrence after index management at our institution was associated with a negative difference in median OS (57.1 months [for no recurrence] vs 31.0 months), highlighting the aggressive biological nature of these cancers. Ninety percent of recurrences were local in nature, while there was only 1 incidence of posttreatment nodal and pulmonary metastases in a patient with SCC. Of the 10 patients who experienced recurrence, 6 underwent further treatment, while 4 patients refused therapy. For patients with bulky disease local to the original surgical cavity, further resection was pursued in addition to chemotherapy (4 patients). For patients with inoperable recurrences (one patient with tumor in the cavernous sinus and another with 
tumor in the oropharynx), chemotherapy alone was pursued. It is notable that there was a statistically significant difference in median OS (67.2 vs 14.4 months, $\mathrm{p}<0.001)$ between those patients who underwent treatment for recurrent disease versus those who did not. While this could be reflective of a treatment bias, these data do potentially underscore the impact treatment has on the natural history of this disease.

\section{Surgical Factors Affecting PFS and OS}

The multidisciplinary management of advanced nonmelanoma cutaneous cancers invading the intracranial space is associated with several challenges. There are numerous considerations with regard to the multidisciplinary management of this disease.

Advanced tumor stage and the extent of intracranial extension negatively impacted patient outcomes. According to the TNM classification, T4 stage was associated with a clear decrease in median PFS (21.4 vs 91.2 months), confirming the prognostic value of the staging system. Interestingly, no particular anatomical compartment in the skull base (i.e., ethmoid sinus, infratemporal fossa, and temporal bone) was associated with a poor PFS or OS. Prior studies of other malignancies have demonstrated the negative correlation between location and OS, such as with skin cancers with their epicenter in the external auditory meatus. ${ }^{3}$ These data support skull base resection regardless of the anatomical compartment involved as long as negative margins can be safely obtained. Invasion of the brain portends a poor outcome, being uniformly fatal past 1 year. It is important to note that other studies have demonstrated anecdotal numbers of long-term survivors with brain invasion from advanced SCC after temporal bone resection. ${ }^{10}$ Hence, resection in this situation cannot be uniformly recommended. Rather, a case-by-case evaluation of the patient's palliative goals is necessary.

A trend toward decreased survival was noted with perineural invasion with a median OS of 31 months (vs 53.8 months) and median PFS of 91 months (vs 105.4 months). It should be noted, however, that the presence of perineural involvement was not prohibitive to obtaining negative margins; this was still achieved in $47 \%$ of those with perineural involvement. Hence, a role for surgery remains in patients with cranial nerve involvement when negative margins can be obtained. In a cohort of 31 patients with cutaneous head and neck SCCs with clinical perineural invasion, Panizza et al. similarly demonstrated that appropriately planned surgery designed to obtain margins along diseased nerve segments improved survival. ${ }^{11}$ Most commonly, branches of CNs V and VII are involved and perineural invasion occurs in the form of gross disease evident radiographically or microscopic infiltration noted on intraoperative margins. In managing trigeminal involvement, our belief is that margins can be safely obtained as far distal as the gasserian ganglion. Depending on the extent of intracranial disease, this can be done via a transfacial approach for minimal extension or via the addition of a craniotomy when significant intracranial disease exists. For CN VII invasion, transpetrosal approaches can be designed to also obtain margins along the facial nerve up to the geniculate ganglion.
Similarly, cavernous sinus invasion was associated with a slight decrease in median OS (43 vs 31 months). There are a fraction of patients who can undergo a lateral cavernous sinus dissection (i.e., gasserian ganglion) to obtain margins with minimal risk of vascular injury or ocular morbidity from injury to the cranial nerves. In the 6 patients with cavernous sinus involvement, disease was cleared in this region in 3 patients. In patients undergoing this dissection without a radical orbitectomy, no issues with ocular anesthesia or mobility were encountered. For the subset of patients with positive margins despite maximal safe resection, we have found radiation therapy to be beneficial for local control. For those patients receiving postoperative radiotherapy as part of the overall treatment plan, the fields are designed to include known sites of positive margins. Our analysis indicates a positive impact on OS when radiotherapy is added (57.1 vs 4.1 months) in situations of cavernous sinus involvement, further indicating that, when part of a multidisciplinary regimen, surgery can be considered in those with cavernous sinus extension.

The highly invasive nature of the disease is highlighted by the 13 patients (54\%) who required radical orbitectomy for orbital disease. The disfiguring nature of this procedure and its impact on quality of life draw its necessity into question. However, on the other hand, untreated disease within the orbit can be a source of pain, proptosis, exposure keratitis, corneal abrasions, and poor cosmesis. Unfortunately at this point, effective organ-sparing neoadjuvant chemotherapy protocols do not exist for NMSCs. It is evident that surgery does confer some benefit with regard to oncological control as those patients undergoing radical orbitectomy experienced a median survival of 54 months (vs 31 months).

\section{Study Limitations}

While this study does shed light on factors affecting treatment outcomes, it is limited by its retrospective design and limited cohort size. The extremely limited study population prevented a multivariate analysis; additionally, several of the analyses regarding the impact of treatment factors, such the utility of postoperative radiotherapy, were not adequately powered. The small number of these cases at any one institution highlights the need for larger, welldesigned multiinstitutional studies. Additionally, if possible, it would have been preferable to perform a well-powered analysis for separate histologies as individual cohorts given the different biological behavior. One could also argue that this cohort of patients was highly selected for based on the indications for surgery. Hence, it is difficult to extrapolate these findings to those patients with advanced NMSCs invading the skull base who also have evidence of regional or systemic disease at presentation. For these patients, further studies regarding the impact of neoadjuvant therapy with or without surgery for local control will be necessary. This study can only address the impact of therapy on OS and locoregional control in those patients presenting solely with local disease.

\section{Conclusions}

Advanced NMSCs of the head and neck with skull base and intracranial invasion represent a difficult disease pro- 
cess with relatively little evidence to guide decision making specific to this cohort of patients. We retrospectively reviewed our experience over a 19-year period. While this study was limited by it retrospective design and small cohort size, the data support the role of safe aggressive resection in improving local control and OS. The data demonstrate that once local control is obtained, patients are likely to die of other causes. Surgical considerations that affect outcome include perineural disease, cavernous sinus involvement, and soft-tissue reconstruction. Ultimately, along with improvements in surgical technique, it is the increasing use of postoperative radiotherapy that positively impacts long-term outcomes.

\section{References}

1. Backous DD, DeMonte F, El-Naggar A, Wolf P, Weber RS: Craniofacial resection for nonmelanoma skin cancer of the head and neck. Laryngoscope 115:931-937, 2005

2. Betti R, Crosti C, Ghiozzi S, Cerri A, Moneghini L, Menni S: Basosquamous cell carcinoma: a survey of 76 patients and a comparative analysis of basal cell carcinomas and squamous cell carcinomas. Eur J Dermatol 23:83-86, 2013

3. Bhayani MK, Yilmaz TA, Sweeney A, Calzada G, Roberts DB, Levine NB, et al: Sinonasal adenocarcinoma: A 16-year experience at a single institution. Head Neck 36:1490-1496, 2013

4. Clayman GL, Lee JJ, Holsinger FC, Zhou X, Duvic M, ElNaggar AK, et al: Mortality risk from squamous cell skin cancer. J Clin Oncol 23:759-765, 2005

5. Ducic Y, Miles BA, Sabatini P: Extending the traditional resection limits of squamous cell carcinoma of the anterior skull base. Otolaryngol Head Neck Surg 137:899-905, 2007

6. Kropp L, Balamucki CJ, Morris CG, Kirwan J, Cognetta $\mathrm{AB}$, Stoer CB, et al: Mohs resection and postoperative radiotherapy for head and neck cancers with incidental perineural invasion. Am J Otolaryngol 34:373-377, 2013

7. Lewis CM, Glisson BS, Feng L, Wan F, Tang X, Wistuba II, et al: A phase II study of gefitinib for aggressive cutaneous squamous cell carcinoma of the head and neck. Clin Cancer Res 18:1435-1446, 2012

8. Lin C, Tripcony L, Keller J, Poulsen M, Dickie G: Cutaneous carcinoma of the head and neck with clinical features of peri- neural infiltration treated with radiotherapy. Clin Oncol ( $R$ Coll Radiol) 25:362-367, 2013

9. Maghami EG, Talbot SG, Patel SG, Singh B, Polluri A, Bridger PG, et al: Craniofacial surgery for nonmelanoma skin malignancy: report of an international collaborative study. Head Neck 29:1136-1143, 2007

10. Moffat DA, Wagstaff SA, Hardy DG: The outcome of radical surgery and postoperative radiotherapy for squamous carcinoma of the temporal bone. Laryngoscope 115:341-347, 2005

11. Panizza B, Solares CA, Redmond M, Parmar P, O'Rourke P: Surgical resection for clinical perineural invasion from cutaneous squamous cell carcinoma of the head and neck. Head Neck 34:1622-1627, 2012

12. Panje WR, Ceilley RI: The influence of embryology of the mid-face on the spread of epithelial malignancies. Laryngoscope 89:1914-1920, 1979

13. Papadopoulos O, Konofaos P, Chrisostomidis C, Champsas G, Frangoulis M, Karakitsos P, et al: Nonmelanoma skin tumors involving the craniofacial region: our 22 years of experience. J Craniofac Surg 18:1021-1033, 2007

14. Rosenthal DI, Harris J, Forastiere AA, Weber RS, Ridge JA, Myers JN, et al: Early postoperative paclitaxel followed by concurrent paclitaxel and cisplatin with radiation therapy for patients with resected high-risk head and neck squamous cell carcinoma: report of the phase II trial RTOG 0024. J Clin Oncol 27:4727-4732, 2009

\section{Author Contributions}

Conception and design: DeMonte, Raza. Acquisition of data: Raza, Ramakrishna. Analysis and interpretation of data: DeMonte, Raza, Weber, Kupferman, Gidley, Hanna. Drafting the article: all authors. Critically revising the article: DeMonte, Raza, Weber, Kupferman, Gidley, Hanna. Reviewed submitted version of manuscript: all authors. Approved the final version of the manuscript on behalf of all authors: DeMonte. Statistical analysis: Raza. Study supervision: DeMonte.

\section{Correspondence}

Franco DeMonte, Department of Neurosurgery, The University of Texas MD Anderson Cancer Center, 1515 Holcombe Blvd., Unit 442, Houston, TX 77040-4009. email: fdemonte@mdanderson. org. 